\title{
Preparación y organización doméstica para las negociaciones del TLC Perú - EE.UU.
}

\author{
Julio Chan Sánchez*
}

\section{RESUMEN}

Este trabajo presenta el proceso de preparación para las negociaciones del acuerdo de libre comercio del Perú con Estados Unidos, así como el proceso de coordinación interna para afrontarlas exitosamente y para lograr el apoyo de los actores domésticos relevantes.

Se argumenta que las actividades de organización de preparación, organización, coordinación e información fueron un elemento fundamental para el éxito de estas negociaciones con nuestro principal socio comercial del momento y que fue el primer acuerdo de libre comercio negociado fuera de América Latina.

Palabras clave: negociaciones comerciales internacionales, TLC Perú - EE.UU., Mincetur, acuerdos de libre comercio, actores domésticos.

Domestic preparation and orgatization towards the negotiations of the Peru-USA Free Trade Agreement

\section{Abstract}

This article deals with the preparation process for the negotiations of the Free Trade Agreement between Peru and the United States. It also deals with the internal coordination process developed in order to successfully negotiate with the United States, as well as to obtain support of the relevant domestic actors.

It is argued that the activities related to preparation, organization, coordination and information were a fundamental element for the success of these negotiations with our most important trade partner of the time, being also the first free trade agreement negotiated outside Latin America.

Keywords: international trade negotiations, Peru-USA FTA, Mincetur, free trade agreements, domestic actors.

\footnotetext{
" Coordinador general del Foro de Cooperación Económica Asia-Pacífico, del Ministerio de Comercio Exterior y Turismo (Mincetur), Lima, Perú. PhD. en Ciencia Política, economista. Correo electrónico: jchan@mincetur.gob.pe 
En este trabajo se presenta una visión panorámica del proceso que se llevó a cabo para las negociaciones del Tratado de Libre Comercio con Estados Unidos ${ }^{1}$. La relevancia de este se debe a las siguientes razones. Primero, estas negociaciones fueron las primeras, fuera de América Latina, para obtener para las exportaciones peruanas el acceso a mercado con beneficios arancelarios permanentes. Segundo, estas primeras negociaciones se llevan a cabo con el socio comercial tradicional más importante del Perú que era ya hasta ahora es, además, la superpotencia global ${ }^{2}$. Tercero, las negociaciones son el inicio de la puesta en práctica de la política de Estado de libre comercio básicamente a través de un proceso negociador. Cuarto, son el inicio de una serie de exitosas negociaciones de acuerdos de libre comercio con otros países y grupos de países fuera y dentro de la región. Quinto, lo anterior ha permitido que las negociaciones comerciales internacionales sean parte de la agenda de la política de comercio exterior y de la política económica del país ${ }^{3}$, así como afianzar, en el ámbito interno el apoyo, por parte de los actores domésticos y de la población al enfoque de economía abierta al mundo que es la política de Estado que el Perú está siguiendo desde principios de la década de los noventa del siglo pasado. Sexto, las negociaciones comerciales internacionales ya no constituyen un tema de debate público acalorado, sino sobre todo una información que es recibida con beneplácito por los actores domésticos y el público en general, sin que falten algunas voces minoritarias con argumentos contrarios, pero casi siempre en asuntos muy específicos.

En esta visión panorámica, primero se hace una breve reseña de los antecedentes mediatos e inmediatos de las negociaciones del tratado de libre comercio Perú Estados Unidos. Luego se reseña el proceso desarrollado por el Perú para enfrentar exitosamente estas negociaciones en el ámbito doméstico durante las negociaciones para negociar el tratado de libre comercio, luego del anuncio de Estados Unidos del inicio de las negociaciones y durante las negociaciones mismas. Finalmente, se presentan algunas reflexiones.

\footnotetext{
1 Este trabajo es una ampliación y profundización que sobre las negociaciones comerciales internacionales son abordadas en el artículo en revisión: Política exterior del Perú en el siglo XXI y las órdenes regionales y hemisféricas. Del mismo se han tomado algunos extractos así como otros han sido ampliados y profundizados.

2 Esto en contraposición de otros Estados que han preferido iniciar negociaciones de tratados de libre comercio no con su principal socio o algún socio importante, sino con socios menores. Tal es el caso de Corea del Sur cuya primeras negociaciones comerciales fuera de su región fue con Chile. Este proceder, sin embargo, parece ser más frecuente que el camino seguido por el gobierno peruano.

3 «El mérito a inicios del presente siglo fue elaborar una agenda comercial que no existía y colocarla en la agenda política y económica del país» (Ferrero, 2013, p. 121).
} 


\section{Situación de las negociaciones comerciales internacionales del Perú antes de negociar el tratado de libre comercio con Estados Unidos}

La participación del Perú en las negociaciones comerciales internacionales puede rastrearse desde las últimas décadas del siglo XVIII (Chan, 1996, pp. 38-39). Sin embargo, es solo en el siglo XXI en el que el gobierno peruano implementa, como política de Estado, la integración comercial con el mundo por medio de negociaciones de acuerdos de libre comercio.

A partir de inicios de la década de los noventa del siglo pasado el Perú volvió a cambiar radicalmente su política económica. Se regresa nuevamente al modelo de desarrollo económico basado en el libre mercado, con su corolario de promover una economía abierta al mundo, es decir de comercio internacional basado en las ventajas comparativas. Estos lineamientos generales de política han sido implementados, desde entonces, a través de desarrollos que el sector del Poder Ejecutivo responsable ha diseñado y perfeccionado a través del tiempo y las experiencias. Sin embargo, cabe resaltar que esta política de Estado de libre comercio en el Perú no es nueva ${ }^{4}$. Lo que la hace especial o diferente en el siglo XXI es que esta política de libre comercio se está logrando fundamentalmente por medio de negociaciones en base a las circunstancias y posibilidades domesticas e internacionales y no a través de la apertura unilateral de la economías.

Esta apertura negociada, geográficamente ya no se centra en los países limítrofes ni tampoco, más ampliamente en la región latinoamericana ni en el continente europeo. Desde mediados de la década de 1990 se prioriza el fortalecimiento de las relaciones con Estados Unidos y sobre todo el acercamiento comercial y financiero con el Este del Asia, particularmente con la China.

4 El libre comercio ha sido la política de comercio exterior peruano durante la mayor parte de la vida independiente del Perú coincidente, hasta cierto punto, con el auge de las exportaciones, la estabilidad política, el conservadurismo social y en el ámbito internacional con auge y niveles de creciente interdependencia económica y política. Sin embargo, sobre todo por circunstancias domésticas, hasta el momento el país no ha podido capitalizar los periodos de auge gracias a la bonanza exportadora y sentar las bases de un crecimiento sostenido y por ende un camino al desarrollo y mayor bienestar generalizado (Thorp y Bertram, 1988; Parodi, 2003; 2016, Sheahan 2001).

5 Sin embargo, es fundamental reiterar que esta condición posiblemente necesaria, no es de ninguna manera suficiente para la mejora general del bienestar de la población. Es necesario, además de otras políticas, que se aprovechen cada vez más y mejor las oportunidades que logran los negociadores al obtener acceso de nuestros productos con beneficio. Ello implica una mayor producción de mercancías con ventajas comparativas y competitivas, así como también, y en base a estas ventajas, desarrollar variaciones de los mismos productos para darles mayor valor agregado así como la producción de productos nuevos. Todos ellos deben aprovechar eficientemente los múltiples, variados y abundantes recursos humanos y naturales que tenemos.

Asimismo, según Dingemans (2013, p. 150), es esencial que la política de negociaciones comerciales internaciones, como parte de la política de comercio exterior, se haga más estratégica. Ello implica que la política comercial, deba estar articulada con las otras políticas de Estado así como con otros sectores de la economía y de la sociedad. 
A nivel mundial, las negociaciones para la finalización de la Ronda Uruguay ${ }^{6}$ y el establecimiento de la Organización Mundial del Comercio (OMC), en enero de 1995, marcan la intensificación de las negociaciones comerciales internacionales del Perú. Nuestro país participó activamente en las negociaciones de la Ronda Uruguay y se constituyó en uno de los miembros fundadores de la OMC. En la OMC, el Perú participa de manera intensa en las negociaciones de la Ronda de Doha ${ }^{7}$. La ronda fue lanzada en noviembre de 2001 durante la IV Conferencia Ministerial de la OMC, realizada en Doha, Qatar ${ }^{8}$. Considerando las ocho rondas realizadas en la era del GATT, esta primera de la OMC es la más prolongada y aún no tiene visos de finalizar.

Hasta principios del siglo XXI, el Perú solo tenía acceso preferencial a mercados en base a un limitado grupo de acuerdos comerciales negociados. El más importante de ellos era el logrado por el Acuerdo de Cartagena, comúnmente conocido como Grupo Andino o Pacto Andino y actualmente tiene la denominación de Comunidad Andina ${ }^{9}$ que solo en 2005 logró a constituirse en una zona de libre comercio ${ }^{10}$. Otro acceso preferencial a mercados en base a negociaciones fueron los acuerdos parciales (con Chile y con México) rescatados de la transformación de la ALALC en ALADI ${ }^{11}$.

Por otro lado, de manera unilateral, el Perú era beneficiario fundamentalmente de dos mecanismos. Uno era el Sistema Generalizado de Preferencias (SGP) que los países desarrollados del Acuerdo General de Aranceles y Comercio (GATT) otorgaban a los menos desarrollados. Y el otro fue el Acta de de Promoción Comercial

\footnotetext{
6 Fue la octava ronda, la más larga de negociar y la última Ronda de Negociaciones del Acuerdo General de Aranceles y Comercio (GATT, por sus siglas en inglés). La finalidad de estas rondas es avanzar en la liberalización comercial a nivel mundial. La VIII Ronda se inició en Punta del Este (Uruguay) en 1986 y concluyó en Marrakech (Marruecos) en diciembre de 1993. Los acuerdos de la VIII Ronda posibilitaron el reemplazo del GATT por la Organización Mundial del Comercio, véase Mindreau, 2005 para más detalles.

7 La Ronda Doha formalmente se le denomina Programa de Doha para el Desarrollo (DDA).

8 La Conferencia Ministerial es la máxima autoridad de la OMC, se reúne cada dos años y está conformada por los Ministros de Comercio de los 164 miembros.

9 «Durante años la política comercial del Perú estaba basada en una integración con los países de la región, la Comunidad Andina, mercados también pequeńos y pobres» (Ferreyros, 2013, p. 119).

10 Si bien ha tenido avances importantes en otros ámbitos (Del Prado, 2015, pp. 160-163; Milet, 2016, pp. 81-82); los resultados han sido menores a los esperados en relación a esfuerzos desplegados por el Perú en este proceso. Las razones fundamentales son las políticas económicas diferentes y visiones políticas distintas (Chan, 1996; 1997; Palma, 2015, p. 299). Sin embargo, «[...] la permanencia del Perú se explica en gran medida [...] por el beneficio [...] para la pequeña y mediana industria nacional al ser un mercado privilegiado para los bienes con mayor valor agregado" (Del Prado, 2015, p. 171).

11 La ALALC (Asociación Latinoamericana de Libre Comercio) establecida en 1960 para crear, mediante negociaciones anuales, una zona de libre comercio entre sus 11 miembros. Fue reemplazada en 1980 por la ALADI (Asociación Latinoamericana de Integración). Esta diluye los compromisos y elimina los plazos previstos en la ALALC. Ninguno de estos dos proyectos integracionistas latinoamericanos han podido tener avances relevantes debido a que los mayores beneficiados eran los miembros más desarrollados: Argentina, Brasil y México (Chan, 1996, pp. 51-57).
} 
Andina (Andean Trade Preference Act - ATPA) de diciembre 1991 12 , de diez ańos de duración. En ambos casos, el acceso era a un limitado número de productos. En el primero de los nombrados, además, sus reglas y los miembros beneficiados podían cambiar año a año y en el segundo, su futuro era incierto luego de los diez años.

En otras palabras, hasta fines del siglo XX, el Perú solo había negociado acuerdos que contenían aspectos relacionados con el acceso preferencial de productos a mercados de la subregión andina y de la región latinoamericana.

A partir de julio de 2001, con el gobierno elegido luego de la transición gubernamental, se reiteraron las líneas maestras de la política de crecimiento económico del país. La economía de mercado en el ámbito interno, y la economía abierta al mundo en el ámbito internacional. Más aún, en los círculos del ejecutivo se va a afianzando la concepción de las exportaciones como un motor de crecimiento. Para ello, se va perfilando el mecanismo de las negociaciones comerciales como uno de los instrumentos que lleve a una mayor integración comercial con el mundo y dando lugar a mayores oportunidades de negocios a las empresas.

Desde el ámbito institucional, el gobierno dio pasos concretos y realizó los ajustes necesarios para la intensificación de las negociaciones comerciales internacionales. Así, se establece el Ministerio de Comercio Exterior y Turismo (Mincetur) en 2002 (Ley 27790 del 23 de julio 2002), proveniente del ex Ministerio de Industria, Turismo, Integración y Negociaciones Comerciales Internacionales (Mitinci). Posteriormente, se decide en 2007 la consolidación de la Comisión para la Promoción de Exportaciones (Prompex) ${ }^{13}$ en la Comisión de Promoción del Perú para la Exportación y el Turismo (PromPerú) ${ }^{14}$. Posteriormente, para el mejor y mayor aprovechamiento de las oportunidades comerciales que se originen de los acuerdos de libre comercio, se lleva a cabo la reestructuración del Mincetur en 2015. ${ }^{15}$ Adicionalmente, las Oficinas

12 El Acta tenía como objetivo promover la producción y exportación de sustitutos de la coca en el Perú, el Ecuador, Bolivia y Colombia, para apoyar a su erradicación y a la reducción del narcotráfico.

13 Establecida en 1996 teniendo como presidente al Primer Vicepresidente de la República. Con la creación el Mincetur, Prompex fue adscrita a dicho nuevo ministerio (Guillen 2004).

14 Encargada también de promover la imagen del país y más recientemente de las inversiones.

15 Por Decreto Supremo 005-2002-MINCETUR del 28 de agosto de 2002, se aprueba el Reglamento de Organización y Funciones del Mincetur. En el mismo se determina que los Órganos Dependientes del Viceministerio de Comercio Exterior eran las Direcciones Nacionales de:

- Integración y Negociaciones Comerciales Internacionales

- Asuntos Multilaterales y Negociaciones Comerciales Internacionales

Desarrollo de Comercio Exterior

- Descentralización de Comercio y Cultura Exportadora

Además se contaba con la Oficina de Estudios Económicos como un órgano de asesoramiento.

Esta estructura es modificada el 12 de junio de 2015 por el Decreto Supremo 002-2015-MINCETUR. Con ello se le está dando mayor énfasis al aprovechamiento de los mercados preferenciales e implementar los compromisos adquiridos en los TLC (García, 2015, pp. 140-142). En este Decreto se establecen las Direcciones Generales de 
Comerciales en el Exterior (OCEX), por Ley 30114, desde 2014, pasaron a depender financiera y funcionalmente del Mincetur. ${ }^{16}$

La primera negociación comercial del Perú en el siglo XXI fue la de la transformación del Acta de Promoción Comercial Andina (Andean Trade Preference Act - ATPA $)^{17}$ en el Acta de Promoción Comercial Andina y Erradicación de Droga (Andean Trade Preference and Drug Erradication Act - ATPDEA). Ante el vencimiento de la vigencia del ATPA, a principios de 2001, el Mincetur concluye que aunque el aprovechamiento del ATPA por parte de los exportadores peruanos había sido importante pero limitado, era necesario negociar su prórroga. Sin embargo, los funcionarios encargados de renegociar el ATPA, en una acción y propuesta muy audaz en esos momentos, lograron convencer primero al viceministro y luego, junto con el viceministro, al ministro, de que además se debía negociar para lograr que los beneficios se amplíen a productos que el Perú estaba en condiciones de exportar con ventajas comparativas.

Tomada la decisión, funcionarios del entonces Viceministerio de Integración y Negociaciones Comerciales Internacionales, inician la labor, primero de convencer de dicha necesidad a los empresarios peruanos para lograr su apoyo. Esta decisión fue prontamente apoyada por las asociaciones empresariales, sobre todo por los gremios exportadores. La segunda actividad constituyó en convencer a los otros tres socios andinos beneficiarios de ATPA (Bolivia, Colombia y Ecuador) de renegociar conjuntamente. Finalmente, el tercer paso fue negociar los cuatro países beneficiarios con EE.UU. Las negociaciones fueron lideradas por el Mincetur que tuvo una actuación importante en la formulación de posiciones comunes de los países andinos en las negociaciones con EE.UU.

El ATPDEA entra en vigencia en octubre de 2002, con efectos retroactivos a inicios de dicho año. Por otro lado, se amplía el acceso con beneficios arancelarios a 700 productos más, pasan de 5600 a 6300, incluyendo muchos productos de interés del Perú, entre ellos, diversos tipos de calzados, textiles y confecciones. La vigencia del ATPDEA fue hasta 29 de febrero de 2008 y se renovó varias veces hasta diciembre de 2010 con la finalidad que los beneficios arancelarios se mantengan en tanto se

\footnotetext{
- Negociaciones Comerciales Internacionales

- Políticas de Desarrollo de Comercio Exterior

- Facilitación del Comercio

- Investigación y Estudios sobre el Comercio Exterior

- Gestión Jurídica Comercial Internacional.

16 Mediante la Ley 29890 de junio de 2012, se transfiere la competencia sobre las Oficinas Comerciales en el Exterior del Ministerio de Relaciones Exteriores al Ministerio de Comercio Exterior y Turismo.

17 El ATPA vencía en diciembre de 2001, contenía acceso sin aranceles para alrededor de 5.600 productos que no incluían textiles, confecciones ni calzado.
} 
finalizaba el proceso de entrada en vigencia del Tratado de Libre Comercio del Perú con Estados Unidos.

De manera casi paralela se llevaba a cabo el proceso de negociación el Área de Libre Comercio de Las Américas (ALCA). En diciembre de 1994 se lleva a cabo la Primera Cumbre de las Américas. Esta trascendental reunión fue convocada por EE.UU. y tuvo como sede la ciudad de Miami. En este evento, los treintaicuatro presidentes invitados (todos los de América y el Caribe menos Cuba) acuerdan, entre otros, las negociaciones del ALCA. En la Segunda Cumbre, Santiago de Chile, abril de 1998, se iniciaron formalmente las negociaciones. En la Tercera Cumbre, Quebec, abril 2001, los presidentes acordaron que las negociaciones culminarían en enero de 2005 y la entrada en vigor del acuerdo sería diciembre de 2005. En Monterrey, Nuevo León, México, en enero de 2004, se realiza una Cumbre Extraordinaria en la cual se expresa explícitamente el disenso solitario de Venezuela en relación al ALCA en una notal al final del texto de la declaración ${ }^{18}$. La Cuarta Cumbre, Mar del Plata, noviembre de 2005; finalizó sin culminar las negociaciones del ALCA. Más aún se tuvo que adoptar la Declaración Presidencial con disonante sobre el ALCA que fue liderada por Venezuela, Brasil y Argentina ${ }^{19}$, que en términos prácticos paralizaron el proceso de la formación del Área de Libre Comercio de las Américas ${ }^{20}$.

\footnotetext{
18 «Venezuela se reserva el párrafo relativo al ALCA, por motivos principistas y diferencias profundas acerca del concepto y la filosofía contenidas en el modelo propuesto, así como por el tratamiento dado a las materias específicas y a los plazos establecidos. Ratificamos nuestro compromiso con la consolidación de un bloque regional y de comercio justo, como base para fortalecer los niveles de integración. Este proceso debe considerar las especificidades culturales, sociales y políticas de cada país; la soberanía y constitucionalidad; el nivel y tamaño de sus economías para garantizar un trato justo» (Cumbre Extraordinaria de las Américas, 2004, pp. 3 y 13).
}

19 Párrafos 19, 19A, 19B:

«19. Reconociendo la contribución que la integración económica puede efectuar al logro de los objetivos de la Cumbre de crear trabajo para enfrentar la pobreza y fortalecer la gobernabilidad democrática:

A. Algunos miembros [propuesta de Panamá, apoyado por EEUU y secundado por la mayoría de los países] sostienen que tengamos en cuenta las dificultades que ha tenido el proceso de negociaciones del Área de Libre Comercio de las Américas (ALCA), y reconozcamos la contribución significativa que los procesos de integración económica y la liberalización del comercio en las Américas pueden y deben aportar al logro de los objetivos de la Cumbre de crear trabajo para enfrentar la pobreza y fortalecer la gobernabilidad democrática. Por ello, mantenemos nuestro compromiso con el logro de un Acuerdo ALCA equilibrado y comprensivo, dirigido a la expansión de los flujos comerciales y, en el nivel global, un comercio libre de subsidios y de prácticas que lo distorsionen, con beneficios concretos y sustantivos para todos, teniendo en cuenta las diferencias en el tamańo y nivel de desarrollo de las economías participantes, y las necesidades especiales y el tratamiento especial y diferenciado de las economías más pequeñas y vulnerables. [ ...]

Instruimos a nuestros responsables de las negociaciones comerciales a reanudar sus reuniones en el curso del ańo 2006, para examinar las dificultades del proceso ALCA, a fin de superarlas y avanzar en las negociaciones, [...] [Las negociaciones no se reanudaron].

B. Otros miembros [propuesta de Venezuela, Brasil y Argentina] sostienen que todavía no están dadas las condiciones necesarias para lograr un acuerdo de libre comercio equilibrado y equitativo, con acceso efectivo de los mercados, libre de subsidios y prácticas de comercio distorsivas y que tome en cuenta las necesidades y sensibilidades de todos los socios, así como las diferencias en los niveles de desarrollo y tamaño de las economías.

En función de lo expuesto hemos coincidido en explorar ambas posiciones [...] [acción que no se llevó a cabo]» (Cuarta Cumbre de las Américas, 2005, pp. 4-5).

20 Pero las Cumbres se siguen llevando a cabo. La VIII se realizó el 13-14 de abril de 2018 en Lima, la primera que se realiza en el Perú. El tema fue «Gobernabilidad democrática frente a la corrupción». La renuncia del presidente 
El Perú, en todo el proceso negociador del ALCA, participó muy activamente en sus rondas y labores relacionadas, defendiendo sus intereses y protegiendo sus sensibilidades y siempre a la búsqueda de consensos constructivos en base a sus lineamientos de política de comercio exterior de integración comercial con el mundo. Al interior del Mincetur, se constituyó un grupo coordinador encargado del liderazgo nacional de estas negociaciones. El grupo, además de las labores logísticas de participación en las reuniones en otros países, y de organización de las mismas cuando el Perú fue el anfitrión, fue responsable de trabajar con las instituciones públicas y privadas correspondientes con la finalidad de definir la posición negociadora nacional en los múltiples asuntos comprendidos en los diversos capítulos que se negociaron. Sin embargo, como ya se ha mencionado, los esfuerzos peruanos no culminaron positivamente debido a las orientaciones de políticas divergentes entre grupos de países participantes.

\section{Argumentando la necesidad del TLC Perú - EE.UU.}

El acceso con beneficios a nuestro principal mercado en esos ańos, era pues precario. Los beneficios unilaterales del ATPDEA, como era el caso del ATPA, estaban sujetos a la certificación anual por parte de la Oficina del Representante de Comercio de Estados Unidos (United States Trade Representative - USTR) de que el Perú estaba cumpliendo con sus compromisos de erradicación del cultivo de la coca. Además tenía a diciembre de 2006 como fecha de finalización ${ }^{21}$ y por ende de renegociación, si hubiese concurrencia para ello. Por otro lado, ya antes de que el ATPDEA reemplazase al ATPA, en 2002, los negociadores del Mincetur, eran conscientes que las negociaciones hacia el ALCA estaban haciéndose cada vez más complicadas, al incrementarse la oposición al mismo sobre todo por parte del gobierno venezolano.

En algún momento, en 2002, finalizadas las negociaciones del TPDEA y antes de su puesta en vigencia, funcionarios del Mincetur encargados de negociar el ATDEA, llevaron a cabo reuniones con sus colegas donde explicitaron la precariedad del acceso de los productos peruanos a su principal mercado. El camino a continuar para superar esta permanente situación de incertidumbre, proponían ellos, era el de negociar un acuerdo de libre comercio.

Las primeras reacciones de los otros funcionarios del Viceministerio de Comercio Exterior fueron argumentos centrados principalmente que el cuestionamiento de

\footnotetext{
Pedro Pablo Kuczynski a fines de marzo de 2018, y su reemplazo por el primer vicepresidente, Martín Vizcarra, conforme a los preceptos prescritos en la Constitución dieron, circunstancialmente, una muchísima mayor relevancia al tema central de esta VIII Cumbre.

21 Esta fue la fecha original de caducidad del ATPDEA.
} 
la necesidad de otorgar concesiones de acceso preferencial al mercado peruano a productos estadounidenses, cuando ya el ATPDEA nos otorgaba acceso preferencial a prácticamente todos los productos exportables peruanos sin dar contraprestaciones.

Dichos funcionarios argumentaban que un tratado de libre comercio (TLC), por el cual el Perú debería otorgar concesiones arancelarias a las exportaciones de Estados Unidos, daría permanencia y seguridad de acceso de nuestros productos al mercado de dicho país ampliando el universo de posibilidades de exportaciones así como incluyendo a los servicios ${ }^{22}$. Más aún, resaltaban la situación que dicho acceso mutuo era mucho más importante para el Perú que para Estados Unidos; ya que para nosotros el mercado de este país era el más importante mientras que no era esta la situación para las exportaciones estadounidenses.

El ATPDEA, tal como estaba negociado, sostenían los funcionarios, mantenía en permanente tensión a los exportadores y a los funcionarios peruanos en relación a si la certificación se otorgaba o no (es decir si se continuaba o no con el acceso preferencial). El TLC daría seguridad y permanencia de esas preferencias que hasta ese momento eran unilaterales. Por ello, el TLC permitiría un planeamiento empresarial adecuado así como también permitiría inversiones y financiamiento para ampliaciones de plantas y el incremento de la producción. Estas inversiones, a su vez, también conllevarían a mejoras tecnológicas, economías de escala y, por ende, mejora de la productividad y competitividad y el desarrollo de nuevas ventajas comparativas y competitivas ${ }^{23}$. Ello, por otro lado, daría la posibilidad de incrementar las exportaciones a EE.UU. en cantidad, valor y variedad con el consiguiente aumento en el empleo formal y la mejorar del bienestar de la población ${ }^{24}$.

22 «El Tratado de Libre Comercio con Estados Unidos (TLC) no solo dará carácter permanente a los beneficios que el ATPDEA - vigente hasta diciembre de 2006- otorga de manera transitoria y unilateral, sino que se ampliará el acceso real a todo el universo arancelario, es decir, permitirá la exportación de todos nuestros productos y servicios a Estados Unidos» (Mincetur, 2005b, p. 8).

«La firma de un tratado de libre comercio (TLC) con Estados Unidos asegurará la permanencia de ese acceso preferencial y potenciará sus beneficios». (Mincetur, 2005a, p. 6).

"Concretar un TLC con los Estados Unidos es prioritario para ampliar y hacer permanente el acceso preferencial que actualmente tienen los productos peruanos al mercado estadounidense a través del ATPDEA». (Mincetur, 2005a, p. 7).

23 «Debido a ello, el TLC constituirá un instrumento valioso de integración que permitirá invertir, planificar y desarrollar nuestras exportaciones en un horizonte de estabilidad, previsibilidad y transparencia» (Mincetur, 2005b, p. 8).

24 «Al ampliar el acceso a mercados y dinamizar el flujo de bienes e inversiones, la integración comercial es uno de los mecanismos más efectivos para impulsar el crecimiento económico y mejorar las condiciones de empleo de la población» (Mincetur, 2005a, p. 9).

«El TLC atraerá un flujo significativo de inversión extranjera directa, con repercusiones favorables en el volumen de exportaciones, la generación de empleo y la transferencia de tecnología» (Mincetur, 2005a, p. 45).

«El acceso preferencial del Perú al mercado estadounidense a través del ATPA primero y luego del ATPDEA ha tenido un impacto significativo sobre las exportaciones y la generación de empleo» (Mincetur, 2005a, p. 6). 
También, estos funcionarios, sustentaban que la reducción de aranceles a las importaciones del Perú procedentes de EE.UU. permitiría a los consumidores una mayor variedad de productos a menor precio, así como a los empresarios a insumos y bienes de capital más variados y a menores precios ${ }^{25}$. Por otro lado, la negociación del primer acuerdo de libre comercio con quien era nuestro principal socio comercial (y superpotencia sin rival hasta el momento), permitiría una experiencia sin igual para proseguir la negociación de otros acuerdo ${ }^{26}$, teniendo en cuenta la imperiosa necesidad del Perú de tener en el incremento de las exportaciones como uno de los motores para el crecimiento ${ }^{27}$, dado el tamaño de nuestra economía ${ }^{28}$.

Adicionalmente, era por todos los funcionarios conocido que, luego del NAFTA, que había entrado en vigencia en 1994, EE.UU. estaba negociando desde hace algunos años un TLC con Chile. Estas negociaciones se habían reanudado con intensidad y que estaba próximo a finalizarse ${ }^{29}$. Asimismo, también era conocido que estaba por iniciarse las negociaciones de un TLC grupal con los países de Centroamérica ${ }^{30}$. La vigencia de TLC de estos países con EE.UU. darían origen a la erosión de las ventajas arancelarias $^{31}$ que el Perú gozaba, ya que dichos países, con estructuras productivas

"Al asegurar este acceso preferencial, el TLC potencia el crecimiento de las exportaciones y la generación de empleos» (Mincetur, 2005a, p. 7).

«El acceso preferencial al mercado estadounidense, a través del TLC, aumentará la demanda de productos peruanos con valor agregado, lo que favorecerá el empleo de trabajadores calificados y la consiguiente mejora en sus ingresos» (Mincetur, 2005a: 13).

«El crecimiento de las exportaciones generado por el ATPDEA está incrementando el empleo formal de manera descentralizada en las regiones. Esto es un claro reflejo de que los beneficios de la apertura comercial no se concentran solo en Lima. El TLC con Estados Unidos generará 81 mil puestos de trabajo adicionales a los ya generados por el ATPDEA» (Mincetur, 2005a, p. 14).

25 «El TLC con Estados Unidos será muy beneficioso para los consumidores peruanos, ya que estos tendrán la posibilidad de escoger sobre la base de una mayor variedad de productos de mejor calidad y con menores precios» (Mincetur, 2005a, p. 8).

26 «El acuerdo comercial firmado con Estados Unidos fue el inicio para el despegue del Comercio Exterior, diríamos el punto de quiebre de la política comercial peruana y la puerta para los demás TLC» (Ferrero, 2013, p. 121).

27 «El TLC es parte de una estrategia comercial integral que busca convertir al Perú en un país exportador, consolidando mercados para nuestros productos, desarrollando una oferta exportable competitiva y promoviendo el comercio y la inversión, para brindar a todos los peruanos mayores oportunidades económicas y mejores niveles de vida» (Mincetur, 2005a, p. 12).

28 «El Perú es un mercado pequeño y con limitada capacidad de compra. Para crecer y producir más, necesitamos de mercados más amplios y con mayor poder adquisitivo" (Ferreyros, 2013, p. 119).

29 Estas negociaciones fueron intermitentes. Toman fuerza a partir de noviembre de 2000. Después de 14 rondas, en diciembre de 2002, son finalizadas. El acuerdo entró en vigencia el 01 de enero de 2004.

30 CAFTA, por sus siglas en inglés. Son partes: Costa Rica, El Salvador, Guatemala, Honduras, Nicaragua y República Dominicana. Las negociaciones se iniciaron en enero de 2003 con los países centroamericanos y en enero de 2004 con la República Dominicana. Las ratificaciones se realizaron entre diciembre de 2004 con El Salvador y octubre de 2007 con Costa Rica, haciéndolo en junio de 2005, EEUU.

31 «De no suscribir un TLC con Estados Unidos, el Perú podría perder los beneficios obtenidos gracias al ATPDEA y quedaría en desventaja comparativa frente a otros países que ya obtuvieron o están en camino de obtener un acceso preferencial al mercado norteamericano» (Mincetur, 2005a, p. 11). 
que compiten con la peruana, tendrían acceso a rebajas arancelarias que eventualmente fuesen igual, y en algunos casos mayores, a las que el Perú tenía y por lo tanto deberían desde entonces competir en igualdad o inferioridad de condiciones arancelarias.

Un argumento fundamental fue que negociar exitosamente con nuestro principal socio comercial del momento y superpotencia sin competencia, daría al Perú varias ventajas en el ámbito internacional. Primero, la primacía del mercado de EE.UU., que siendo nuestro principal socio comercial, hacia obvia la elección de a qué país solicitar la negociación de un TLC ${ }^{32}$. Ello además nos daría mucha experiencia para negociar con más capacidad acuerdos con otras potencias económicas del mundo.

Segundo, el haber abierto nuestro mercado a la producción de EE.UU., incrementaría la competencia interna y promovería una mayor productividad y competitividad de la producción nacional.

Tercero, lo anterior haría más fácil negociar con otros países, en razón a la invalorable experiencia negociadora lograda. Además, debido a que las rebajas arancelarias importantes ya habían sido otorgadas a EE.UU. (con importantes excepciones), no iban a ser problemáticas en otorgarse a su vez a otros países, más aún cuando las empresas peruanas ya habían o estaban mejorando su competitividad ${ }^{33}$.

Cuarto, otorgar similares o iguales condiciones de acceso que las gozadas por EE.UU. a las importaciones peruanas de otros países con TLC permitiría una mayor competencia entre las fuentes de importación (sobre todo de los muchísimos productos de manufactura avanzadas no producidos en el Perú pero fundamentales para los sectores productivos) y diversificación de fuentes de las importaciones peruanas y variedad de productos, con una esperada reducción de los precios, sobre todo de los insumos y de los bienes de capital.

Quinto, un TLC con EE.UU. daría señales al resto del mundo de la importancia y seriedad que tiene la política de comercio exterior del Perú de libre comercio y de integración comercial con el resto del mundo, así como del interés peruano de implementarla en base a sus necesidades e intereses.

Sexto, el acuerdo con EE.UU. mejoraría la imagen y el prestigio nacional a los ojos no solo de los peruanos sino también a los ojos del resto del mundo. Asimismo,

\footnotetext{
32 «El TLC con EE.UU. fue la primera gran experiencia. Y era natural, era nuestro principal socio comercial» (Ferreyros, 2013, p. 119).

33 Adicionalmente, las rebajas arancelarias que el Perú pudiese obtener a través de las negociaciones con otros países, permitiría eliminar o reducir la erosión de su acceso a mercados a dichos países en los casos en que estos países ya hubiesen otorgados rebajas arancelarias debido a TLC en vigencia con naciones que tuviesen estructuras productivas similares a la peruana.
} 
fortalecería la posición del Perú como un socio confiable que promueve el libre comercio a través de regulaciones claras, transparentes y vinculantes.

Sétimo, una exitosa y ejemplar negociación del primer acuerdo de libre comercio peruano con un país fuera de la región latinoamericana, y sobre todo siendo este con EE.UU., daría pie a un círculo virtuoso de negociaciones ${ }^{34}$. Estas negociaciones exitosas darían lugar a oportunidades comerciales que conllevarían un mayor bienestar de la sociedad peruana. Todo ello haría que los empresarios y otros actores directamente relacionados con el comercio exterior apoyasen políticamente estas actividades. Ello en función a que serían percibidas, en balance, como beneficiosas no solo para sus intereses privados y para el gobierno de turno, sino, más importante aún, para los intereses nacionales tanto en el ámbito doméstico como en el internacional.

Estas argumentaciones analizadas, discutidas y desarrolladas entre los funcionarios del Mincetur, fueron a su vez llevadas a los diversos actores directamente involucrados o interesados en el tema. Así, los funcionarios del Mincetur sostuvieron reuniones con líderes y hacedores de opinión de los ámbitos académicos, gremiales profesionales, gremiales empresariales tanto manufactureros como agropecuarios y centros de investigación.

Estas actividades daban a conocer a los actores relevantes el pensamiento al interior del Mincetur a nivel técnico, del posible nuevo instrumento que se adoptaría para promover las exportaciones. También sirvieron para tratar de convencerlos de sus bondades ya que sería un eficaz instrumento de la economía de mercado, la economía abierta, y el incremento de la competencia y de la competitividad. Asimismo, se hacía explicita la necesidad de resolver la situación de precariedad de las exportaciones peruanas a nuestro principal mercado y la posibilidad de acceder a importaciones más baratas como también promover inversiones con financiamiento externo.

El éxito de los diálogos con los actores relevantes, permitieron plantear primero al viceministro, Alfredo Ferrero y luego, a través de él, al ministro Raúl Diez Canseco, la conveniencia de proponer a EE.UU. la negociación de un acuerdo de libre comercio. Los argumentos y contraargumentos fueron similares a los arriba mencionados, lográndose, sin embargo, convencer al primero ${ }^{35}$ y luego obtener el apoyo de las otras altas autoridades del Mincetur. El Ministro, a su vez, llevó la propuesta al Consejo de

\footnotetext{
34 «La política exterior del Perú [...] mantuvo una visión coherente con la interdependencia compleja del sistema internacional, logrando una creciente inserción en la economía global, acelerada a partir del acuerdo de libre comercio con Estados Unidos que, a su vez, mejoró la capacidad negociadora para lograr acuerdos similares con una diversidad de países» (Ramacciotti y Méndez, 2012, p. 136).

35 «Al inicio había incredulidad respecto a los beneficios de los TLC e incluso respecto a la posibilidad de negociar con Estados Unidos» (Ferrero, 2013, p.121).
} 
Ministros, que en varias sesiones del Consejo se avanzó hacia el consenso. De manera paralela, los funcionarios del Mincetur, hacían labor similar con los funcionarios responsables en los ministerios clave como el de Economia y Finanzas y Relaciones Exteriores, así como el de la Presidencia del Consejo de Ministros, de Producción y de Agricultura, entre otros.

En el frente externo, el viceministro de Comercio Exterior, Alfredo Ferrero, aprovechaba cuanta oportunidad tenia para solicitar y obtener una reunión bilateral con el embajador Robert Zoellick, representante de Comercio de Estados Unidos (USTR United States Trade Representative ${ }^{36}$. En la misma, nunca dejaba de proponer la realización de un tratado de libre comercio bilateral.

El representante de comercio estadounidense daba al principio respuestas contundentemente negativas, que luego se tornaron solo negativas y posteriormente en evasivas, para finalmente acceder a la posibilidad de una negociación de un tratado de libre comercio. Sin embargo, sostuvo que este tratado no se negociaría con el Perú solamente, sino con todos los beneficiarios del ATPDEA siempre y cuando fuese contundente de que las negociaciones del ALCA no fuesen a llegar a su destino.

Es así que en noviembre de 2003, el USTR, informa la intención de EE.UU. de negociar un tratado de libre comercio con Bolivia, Colombia, Ecuador y Perú. ${ }^{37}$ Los esfuerzos intensos del viceministro Alfredo Ferrero daban su primer fruto. ${ }^{38}$

36 Estas oportunidades eran varias al año. Dos en los márgenes de las reuniones de foro de Cooperación Económica Asia-Pacífico (APEC): ministros responsables de Comercio, y reunión anual conjunta de ministros de Relaciones Exteriores y de Comercio Exterior (Chan, 2008, pp. 209-210). Además, las reuniones ministeriales y viceministeriales del ALCA, y todas las otras reuniones en las que participaban el viceministro y el USTR.

Asimismo, a nivel presidencial, fue crucial la reunión bilateral entre los presidentes Toledo y Bush (hijo) en los márgenes de la Cumbre de APEC de 2001. Igualmente, fue muy importante la visita del presidente George W. Bush en marzo de 2002, la primera en la historia de un mandatario estadounidense en funciones, donde se volvió a impulsar la negociación del TLC (MRE, s/f).

37 Finalmente, el viceministro Ferrero logró que el acuerdo fuese firmado de manera bilateral. El tratado es formalmente denominado «Acuerdo de Promoción Comercial Perú - Estados Unidos». Su denominación en inglés es The United States-Peru Free Trade Agreement (PTPA). Fue suscrito el 12 de abril de 2006, aprobado por el Congreso mediante Resolución Legislativa 28766, publicada en el Diario Oficial El Peruano el 29 de junio de 2006 y ratificado mediante Decreto Supremo 030-2006-RE, publicado en el Diario Oficial El Peruano el 30 de junio de 2006.

Dado que el Congreso estadounidense aun no lo había aprobado y debido al cambio de composición de la Cámara de Representantes de EE.UU., luego de las elecciones congresales de EE.UU. de noviembre de 2006, se negocia un Protocolo de Enmienda que fue suscrito el 25 de junio de 2007, aprobado por el Congreso mediante Resolución Legislativa 29054, publicada en el Diario Oficial El Peruano el 29 de junio de 2007. El Protocolo de Enmienda fue ratificado mediante Decreto Supremo 040-2007-RE, publicado en el Diario Oficial El Peruano el 3 de julio de 2007. El Tratado y el Protocolo fueron puestos en ejecución mediante Decreto Supremo 009-2009-Mincetur, publicado en el Diario Oficial El Peruano el 17 de enero de 2009. El 01 de febrero de 2009, entró en vigor.

38 Para un amplio recuento de estas negociaciones, su proceso de aprobación en los Congresos respectivos y su implementación, véase Ferrero, 2010. 


\section{Preparaciones para la negociación del TLC con EE.UU.}

Durante 2003, en previsión de las negociaciones del tratado con EE.UU. y basados en las experiencias y negociaciones recientes y concurrentes, sobre todo en la OMC, del ATPDEA y del ALCA, se fue preparando en el Mincetur un plan de actividades destinado al fortalecimiento de capacidades de los funcionarios. En el primer semestre de 2004, las actividades del plan se implementaron. Profesores de la Escuela de Gobierno John F. Kennedy de la Universidad de Harvard impartieron a los funcionarios un seminario intensivo sobre cómo es el proceso de las negociaciones comerciales de EE.UU. En este se aprendió la estructura formal, legal y procedimental de estas negociaciones desde antes de que se hagan públicas hasta su puesta en vigencia. También se tuvo conocimiento de la cultura y estilo de negociación del funcionario estadounidense así como sus principales estrategias, tácticas, y modos de relacionamiento personal y las vinculaciones y relaciones entre los negociadores y los empresarios y los congresistas.

Por otro lado, se invitó a varios negociadores de Chile y de Costa Rica, países que hacía poco habían finalizado sus negociaciones comerciales con EE.UU. Ellos proveyeron abundante información de primera mano y muy actualizadas sobre sus experiencias negociadoras con funcionarios de EE.UU., así como su manejo de sus negociaciones en el ámbito interno. Adicionalmente, se impartieron dos talleres sobre técnicas de negociación.

Con estos conocimientos y habilidades, los funcionarios del Mincetur, en especial el grupo designado para coordinar las negociaciones, desarrollaron un esquema flexible de trabajo. Flexible en el sentido que estuvieron en la capacidad de ajustar, modificar y reformular dicho plan de trabajo y su logística implícita conforme se acumulaban las experiencias negociadoras tanto en el frente interno, como en el frente externo con los otros países andinos y con EE.UU.

En el frente interno se presentaban los siguientes actores a quienes habían que convencer: funcionarios de otros ministerios y organismos públicos responsables de las funciones relacionadas a los asuntos a ser negociados en los diversos capítulos contenidos en el TLC; empresarios industriales potencialmente beneficiados o afectados por las concesiones arancelarias a otorgarse agrupados en sus gremios empresariales; empresarios agropecuarios y trabajadores del campo representados por sus asociaciones respectivas; académicos que analizaban, estudiaban y seguían las negociaciones como un asunto inédito para el país; trabajadores manufactureros agrupados en sus instituciones sindicales; hacedores de opinión pública, empresas de investigación, medios de comunicación masiva y el público en general. 
Una interés especial fue el de involucrar a los miembros provinciales de los actores antes mencionados en reconocimiento de la importancia de que los beneficios fuesen lo más descentralizados posibles y con la finalidad de conocer con profundidad sus principales preocupaciones y temas sensibles.

El Mincetur, para llevar a cabo estas importantes acciones, obtuvo un financiamiento adicional en su presupuesto. Con ello también se pudo ampliar la plana profesional. Se dio preferencia a la contratación de profesionales jóvenes y a recién egresados. Asimismo, se contrataron a expertos en organización y en comunicaciones. Además, parte del incremento presupuestal sirvió para contratar servicios especializados para la preparación, difusión y evaluación de información ${ }^{39}$; y para llevar actividades de difusión en todo el país, tanto las organizadas por el Mincetur, como aquellas a las que se invitaba al Mincetur a participar.

En forma paralela, y poniendo las bases para futuras negociaciones comerciales internacionales, con el fortalecimiento de la Oficina de Estudios Económicos, el Mincetur pudo llevar a cabo una investigación que ha permitido contar con una lista de países con los que hay prioridad y con los que son de interés tener TLC. Ello ha permitido a las altas autoridades proponer TLC con los países prioritarios, y a considerar favorablemente las solicitudes de negociación de TLC de los países tanto prioritarios como de interés. Además, esta área preparó la información sobre la importancia del TLC con EE.UU., así como las fortalezas y debilidades tanto negociadoras como productivas del país.

\section{El proceso interno de coordinación durante las negociaciones del TLC con EE.UU.}

Con el transcurrir de las negociaciones, las acciones de transparencia y de comunicación fueron delineando un procedimiento que dio resultados exitosos. El plan comunicacional y de transparencia fue esencial para el logro del apoyo tanto al proceso negociador como al proceso de ratificación e implementación del acuerdo. No solo de los actores directamente involucrados, sino también de la población en general se obtuvo una rotunda aprobación al proceso de negociación y sobre todo a sus

\footnotetext{
39 Para ello se estableció la Unidad de Comunicaciones para el TLC Perú-Estados Unidos. A cargo de ella, entre otros, estuvo la preparación y publicación de (Mincetur, s/f):

- TLC - nociones clave

- Historieta: las oportunidades del TLC

- Las mipymes frente al TLC

- TLC la oportunidad de hoy

- TLC y agro
} 
resultados, los que a la vez que abría el mercado de Estados Unidos a nuestras exportaciones, se tomaba en cuenta las sensibilidades más importantes del Perú.

Las coordinaciones con el sector público se realizaron directamente entre los funcionarios de las instituciones involucradas en sus niveles respectivos. En estas reuniones se analizaban las propuestas y se trabajaba la posición nacional a ser aprobada por las altas autoridades, cuando fuese el caso.

Mención especial se debe hacer a las estrechas coordinaciones con el Poder Legislativo, concretamente con la Comisión de Comercio Exterior y también con la de Relaciones Exteriores, entre otras. Con los congresistas de dichas comisiones y sus asesores, se realizaron sendas reuniones de trabajo a cargo de las altas autoridades del Mincetur. En las mismas se les mantenía informado de los avances y principales temas con dificultades de acordar entre las partes así como de una prognosis del proceso negociador.

El sector privado, luego de un breve periodo de iniciadas las negociaciones, se organizó en el Consejo Empresarial de Negociaciones Internacionales, CENI. El Consejo presentaba propuestas técnicas y una estrategia negociadora integral y se organizó en mesas de trabajo espejo de las mesas de negociación gubernamentales (García, 2012, p. 452). Este consejo agrupó a los gremios empresariales industriales como agropecuarios, grandes, medianos y pequeños ${ }^{40}$. Además, se llevaron a cabo talleres para involucrar

40 Las instituciones que la conformaron fueron (García, 2012, p. 453):

1. Asociación de Administradoras de Fondos Privados de Pensiones (AAFP)

2. Asociación de Bancos del Perú (ASBANC)

3. Asociación de Empresas de Transporte Aéreo Internacional (AETAI)

4. Asociación de Empresas Privadas de Servicios Públicos (ADEPSEP)

5. Asociación de Exportadores (ADEX).

6. Asociación de Gremios Productores Agroexportadores.(AGAP)

7. Asociación de Representantes Automotrices del Perú (ARAPER)

8. Asociación Peruana de Avicultura (APA)

9. Asociación Peruana de Consultoría (APC)

10. Asociación Peruana de Empresas de Seguros (APESEG)

11. Asociación Peruana de Entidades Prestadoras de Salud (APEPS)

12. Aociación Peruana de Productores de Azúcar (APPAR)

13. Bolsa de Valores de Lima (BVL)

14. Cámara de Comercio de Lima (CCL)

15. Cámara Nacional de Comercio, Producción y Servicios (PERUCAMARAS)

16. Cámara Nacional de Turismo (CANATUR)

17. Cámara Peruana de la Construcción (CAPECO)

18. Consejo de Organizaciones de la Micro, Pequeña y Mediana Empresa del Perú (COMPYMEP)

19. Convención Nacional del Agro Peruano (CONVEAGRO)

20. Red Nacional de Cámaras (RNC)

21. Sociedad de Comercio Exterior (COMEXPERU)

22. Sociedad Nacional de Industrias.(SNI)

23. Sociedad Nacional de Minería, Petróleo y Energía (SNMPE)

24. Sociedad Nacional de Pesquería (SNP)

25. Sociedad Nacional de Seguridad (SNS) 
a las mipymes en el proces $\mathrm{o}^{41}$. Adicionalmente, los funcionarios negociadores tenían reuniones periódicas con funcionarios de los gremios, y de los sindicatos, con académicos y profesionales de centros de investigación y otras instituciones representativas de la sociedad civil ${ }^{42}$, para que conociesen la situación de las negociaciones y emitieran sus puntos de vista sobre asuntos específicos. Estas reuniones, también sirvieron para que los negociadores tuvieran información de primera mano de las características, fortalezas y debilidades de los sectores productivos en general, de las empresas en particular, así como el proceso productivos de aquellos renglones considerados como sensitivos por el gobierno. Asimismo, los encuentros permitieron dar a conocer en forma detallada preocupaciones concretas de los diversos actores institucionales.

El jefe negociador acompañado por los funcionarios del Mincetur responsables de cada tema sostenían también reuniones con los funcionarios de las diversas reparticiones del sector público, con la finalidad que dichos funcionarios tuviesen una visión general de la marcha del proceso negociador. Por otro lado, estos eventos daban la oportunidad para conocer de primera mano los puntos de vista y temas sensibles para las instituciones públicas.

También estos funcionarios realizaban reuniones con editores, periodistas y hacedores de la opinión pública y sus medios de comunicación tanto de Lima como de provincias con la finalidad que ellos a su vez informaran al público en general.

Por otro lado, en atención a la importancia de los posibles efectos descentralizadores, se hizo un esfuerzo especial para llevar a cabo múltiples reuniones al interior del país. En ellas, los funcionarios del Mincetur daban a conocer regularmente la situación de las negociaciones. Además, ello permitía auscultar la evolución de los puntos de vista,

\footnotetext{
41 «Por primera vez en nuestra historia, las micro, pequeñas y medianas empresas (mipymes) intervienen activamente en una negociación comercial internacional. Para estructurar su posición estratégica e identificar la llamada agenda interna para la búsqueda de competitividad, numerosas mipymes participaron en diversos talleres realizados en diferentes ciudades del país» (Mincetur, 2005, p. 11).

42 Tuvieron una participación y contribuciones destacadas las siguientes instituciones (Mincetur, s/f):

- Propuesta Ciudadana, Programa Laboral de Desarrollo - PLADES,

- Asociación de Industriales Madereros y Afines de Loreto.

- Coalición Peruana para la Diversidad Cultural.

- Proterra.

- Instituto Interamericano por la Gobernabilidad, Democracia y Desarrollo contra la Pobreza Horizontes Libres (IIGDDCP).

- Instituto Interamericano de Cooperación para la Agricultura.

- Central Unitaria de Trabajadores CUT.

- Federación de Pescadores Artesanales de Loreto - Feparel.

- Asociación de la Micro y Pequeña Industria Confeccionista de Gamarra Perú (AMPIC Gamarra).

- Foro Salud.

- Comisión Episcopal de Acción Social.

- Consejo Económico y Social para el Desarrollo Nacional.

- Acción Internacional para la Salud.
} 
los sentimientos y la posición de la población visitada. Asimismo, se aprovechaba para dar entrevistas a los medios de comunicación local para difundir más y mejor la situación de las negociaciones.

De manera periódica, el Mincetur contrató servicios de empresas encuestadoras para conocer el ánimo de la población, sobre todo de provincias, respecto a la marcha de las negociaciones. Ello permitió a los hacedores de decisiones del ministerio determinar cursos de acción más adecuados en las negociaciones, así como en las actividades de difusión y de transparencia en los lugares más necesarios ya sea en Lima como en el interior del país.

El objetivo de todo este esfuerzo coordinador y de transparencia en el ámbito doméstico era

[g]enerar un consenso amplio sobre un tema de la trascendencia del Tratado de Libre Comercio (TLC) entre el Perú y Estados Unidos [lo que] supone articular la participación de numerosos actores $y$, en particular, de aquellos con capacidad de contribuir a la formación de opinión pública. [...] Por ello, reconociendo los retos existentes, y respetando las voces discrepantes de diversos grupos de interés, es preciso difundir entre la población los aspectos relacionados al TLC. Pero, por sobre todo, [...], es indispensable aclarar [que] el ingreso preferencial y permanente de nuestros productos al mercado que más compra en el mundo tendrá un impacto benéfico masivo sobre el crecimiento económico y el bienestar de la población en todos los rincones del país (Ferrero, 2005, p. 4).

Además de las múltiples reuniones con los diversos actores domésticos, se realizaron reuniones con los otros tres socios andinos miembros iniciales de la negociación. En dichas reuniones previas a la primera ronda de negociación los cuatro países señalaron sus objetivos generales, sus intereses ofensivos y defensivos, tratándose en todo momento de lograr una posición consensuada.

Iniciadas las negociaciones las coordinaciones andinas adquirieron mayor importancia. Para el Perú era muy importante lograr una posición andina conjunta en sus temas prioritarios. Ello para tener una mayor fuerza y presión en las negociaciones con EE.UU., y para fortalecer las relaciones bilaterales y andinas. Sin embargo, este objetivo fue pocas veces logrado ${ }^{43}$. Al final de las rondas negociadoras, el Perú fue el primer país que suscribió y puso en vigencia su TLC con EE.UU. ${ }^{44}$.

\footnotetext{
43 Eventualmente, primero Bolivia y luego el Ecuador abandonaron las negociaciones. Hubo desinterés de Bolivia y Ecuador (Ramacciotti y Méndez, 2012, p. 126).

44 Colombia en la última ronda no pudo acompañar al Perú en las decisiones finales, alejándose de las negociaciones. Posteriormente, este país, habiendo superado sus impasses domésticos, reanudó sus negociaciones que finalizaron en febrero de 2066, después de 21 meses, 15 rondas y 100 reuniones. En mayo de 2012 entró en vigencia el Acuerdo de Promociones Comerciales entre Estados Unidos y Colombia.
} 
También se llevaron a cabo reuniones preliminares con la contraparte estadounidense para avanzar en las líneas maestras para las negociaciones. Por ejemplo, cuestiones logísticas como cuál país sería la sede de la primera ronda y de las rondas inmediatamente sucesivas, cuándo se llevaría a cabo la primera ronda, cuántos días duraría, si iban a estar presentes altas autoridades o no etc.; cuestiones sustantivas sobre cuáles serían los grandes asuntos a incluirse en las negociaciones, qué capítulos contendría, cuál sería la estructura del tratado y cómo se les denominaría, etc.

Las negociaciones se realizaron en trece rondas, entre el 18 de mayo de 2004 y el 07 de diciembre de 2005. Estas rondas fueron:

\begin{tabular}{|c|l|l|}
\hline Ronda & Fecha & Lugar \\
\hline I & 18 y 19 de mayo de 2004 & Cartagena de Indias - Colombia \\
\hline II & $14-18$ de junio de 2004 & Atlanta - Estados Unidos \\
\hline III & $26-30$ de julio de 2004 & Lima - Perú \\
\hline IV & $13-17$ de septiembre de 2004 & San Juan - Puerto Rico \\
\hline V & $25-29$ de octubre de 2004 & Guayaquil - Ecuador \\
\hline VI & 29 de noviembre - 5 de diciembre de 2004 & Arizona - Estados Unidos \\
\hline VII & 7 -11 de febrero de 2005 & Cartagena de Indias - Colombia \\
\hline VIII & $14-18$ de marzo & Washington D.C. - Estados Unidos \\
\hline IX & $18-22$ de abril de 2005 & Lima - Perú \\
\hline X & 6-10 de junio de 2005 & Guayaquil - Ecuador \\
\hline XI & 18-22 de julio de 2005 & Miami - Estados Unidos \\
\hline XII & 19-23 de septiembre de 2005 & Cartagena de Indias - Colombia \\
\hline XIII & 14-22 de noviembre - 5-7 de diciembre de 2005 & Washington D.C. - Estados Unidos \\
\hline
\end{tabular}

Fuente: Mincetur s/f.

Antes de la primera ronda, los funcionarios negociadores del TLC también llevaron a cabo reuniones en el país con los diversos actores ya mencionados. En ellas se dieron a conocer los avances previos ya acordados con los negociadores de Estados Unidos, las coordinaciones realizadas con los otros socios andinos. También se informó sobre los objetivos generales peruanos esperados a lograrse en las negociaciones, así como los principales aspectos que en ese momento se consideraban sensibles para el Perú, y sobre los cuales se les iba a dar especial atención durante las negociaciones.

Durante las rondas se alentó el acompañamiento de los actores domésticos, tanto de las instituciones del Poder Ejecutivo como del Poder Legislativo, así como del sector privado empresarial como social y académico. Los integrantes que no eran funcionarios negociadores gubernamentales se constituyeron en lo que se denominó 
la Sala de al lado. Al final de cada día de negociación los negociadores informaban a los participantes de los avances, la situación en el momento y las perspectivas para el día siguiente o días siguientes de la ronda.

De esta manera los actores domésticos representantes de los diversos intereses internos obtenían conocimiento prácticamente al momento de lo que estaba sucediendo en las reuniones de la ronda. Ello permitía que dichos actores tuviesen una mayor y mejor comprensión de las negociaciones y de los progresos, retrocesos y dificultades que ser iban presentando, así como la complejidad de las negociaciones tanto tema por tema como sus relaciones cruzadas.

Además de la transparencia que este procedimiento ofrecía; los funcionarios negociadores también recibían sugerencias, comentarios y propuestas directamente de los participantes de la Sala de al lado; que en más de una ocasión resultaron útiles. Ello a su vez, hacía de los integrantes de la Sala de al lado, además de sentirse útiles y participes de las negociaciones, sentirse que tuvieron la percepción de ser contribuyentes al progreso y análisis de los temas negociados, así como tal vez, hasta sentirse que estaban haciendo y siendo parte de la historia junto con los negociadores.

Finalizada cada ronda, en Lima, se convocaba a reuniones informativas por un lado con funcionarios de las instituciones del sector público involucradas y por el otro, con las representaciones de los actores privados. En las mismas se daba a conocer de manera detallada pero enfocada a la audiencia, de lo sucedido en la ronda, así como se presentaba una breve prognosis y las labores futuras. En el primer caso, además de estar presentes los funcionarios que participaron en la ronda en representación de sus instituciones; estaban también presentes funcionarios de alto nivel que no habían asistido a la ronda, así como los funcionarios que trabajaban en el tema, pero no tampoco habían participado en las negociaciones. En el segundo caso, también la convocatoria era general y abierta a todos los interesados en las negociaciones, siempre y cuando hubiesen sido acreditados previamente por alguna institución.

A los medios de comunicación se les convocaba a sendas conferencias de prensa; y a los periodistas y hacedores de opinión se les aceptaba entrevistas así como participar en programas en sus respectivos medios. Por otro lado, el Mincetur enviaba notas de prensa a todos los medios nacionales tanto de Lima como de provincias; así como a los servicios internacionales de noticias que se habían registrado con la Unidad de Comunicaciones para el TLC Perú-Estados Unidos. La difusión de las negociaciones fue amplia. En especial las últimas, que dieron lugar a creciente interés de la población en general y a intensos debates en los medios.

Conforme si iban avanzando en las rondas, los temas se iban consensuando y por ello, había cada vez menos personas que participaban en las mismas, al ser sus temas de 
interés ya acordados. Posiblemente, en algunos casos, estos consensos no fueron los que muchos actores esperaban al principio, pero ciertamente casi todos, si no todos, quedaron conformes con los resultados. Todos, asimismo, fueron testigos y acabaron con el convencimiento de que los negociadores habían hecho todos los esfuerzos para lograr el mejor acuerdo posible en sus temas de interés, y habían obtenido la experiencia de haber conocido de primera mano el difícil proceso de llegar a acuerdos, así como la satisfacción de haber participado y contribuido a dichos consensos.

Por otro lado, la comunicación por los medios hacia la población fue siendo cada vez más intensa. Los temas pendientes eran cada vez más complicados de hallarles una solución aceptable a las partes y por otro lado, los actores involucrados en estos temas sensibles hicieron público sus puntos de vista y sus demandas. Entre ellos los temas agrícolas: azúcar, arroz, algodón, maíz y los cuartos traseros de pollo, entre otros ${ }^{45}$. Así como los temas propiedad intelectual: protección a los conocimientos indígenas, acceso a los recursos genéticos ${ }^{46}$, y precio de los medicamentos ${ }^{47}$.

Especial cuidado se tuvo en difundir información y participar en los debates después de la finalización de las negociaciones, en diciembre de 2005, hasta después de su suscripción, en abril de 2006, y durante el proceso de ratificación en el Congreso, en junio de 2007, de la enmienda al tratado. La organización de eventos en provincias por parte del Mincetur y la participación en eventos fuera de Lima organizados por diversas organizaciones locales fueron también esenciales para dar a conocer y para convencer a la ciudadanía de las bondades y ventajas del acuerdo y su protocolo modificatorio, en especial en el rubro agropecuario ${ }^{48}$ y las mipymes. ${ }^{49}$

45 Para estos productos se acordaron beneficios arancelarios solo para cuotas de importación. Además el gobierno se comprometió a tomar las medidas correctivas y de apoyo a los productores agrícolas en el caso de que fuesen afectados negativamente por la implementación del TLC.

"Para hacer frente a los subsidios en el sector agrícola y a los desafíos de la apertura comercial, se viene trabajando en dos vías: la negociación de mecanismos de protección y un Programa Nacional de Reconversión y Compensación para el Agro» (Mincetur, 2005a, p. 26).

Hasta el momento no solo no se han dado estos casos, sino que se han incrementado las ventas agropecuarias al exterior constituyéndose en el sector más importante y dinámico de las exportaciones no tradicionales.

46 En estos casos se llegó a consenso por medio del «Entendimiento con relación a la Biodiversidad y la Protección de los Conocimientos Tradicionales». Por dicho entendimiento se requiere la obtención del consentimiento informado previo al uso de los conocimientos tradicionales y a la distribución justa y equitativa de los beneficios derivados del uso de los mismos con las comunidades indígenas propietarias del conocimiento y/o del recurso (Mincetur, s/fa). 47 El gobierno, durante las negociaciones argumentó que los términos acordados respecto a medicamentos y sus patentes no afectarían los precios de los mismos. Posteriormente el Mincetur informó que de acuerdo a estudios del Ministerio de Salud, luego de la puesta en vigencia del TLC, "[l] os precios de los medicamentos, tanto de marca como genéricos, han bajado de precio. Esto se ha dado debido a la mayor competencia, que favorece al usuario de estos productos" (Mincetur, s/fa).

48 El Mincetur preparó y difundió un documento dirigido a los agricultores. Este fue «TLC y Agro. Todo lo que el productor y el trabajador agrario deben saber acerca del TLC» (Mincetur, 2005c).

49 «Las mipymes frente al reto del TLC. Construcción de una agenda de competitividad desde las bases empresariales» (Mincetur, 2005). 
En varios de estos temas, algunas ONG tuvieron una importante participación directa en el debate público y también en apoyar a organizaciones a articular sus mensajes de oposición así como a realizar la difusión de los mismos. Estas posiciones dieron la oportunidad a las altas autoridades del gobierno de aclarar los términos específicos de los acuerdos incluidos en el TLC respecto a los temas considerados sensibles por dichas organizaciones y, en algunos casos de la población en general o algún importante sector en particular. Estas intervenciones fueron claves para despejar las dudas y para convencer a los actores involucrados que sus preocupaciones habían sido tomadas en cuenta en los resultados de las negociaciones.

A pesar de dichas manifestaciones en contra, la población en general apoyó la adopción del TLC, y el tiempo ha confirmado en los hechos que las preocupaciones de los efectos negativos del mismo fueron básicamente infundadas.

\section{Algunas reflexiones finales}

Las negociaciones comerciales que culminaron con la puesta de vigencia del Acuerdo de Promoción Comercial Perú - Estados Unidos fueron exitosas. Buena parte de este éxito se puede atribuir a la organización interna para llevar a cabo el proceso de negociaciones que el Mincetur diseñó y puso en práctica.

El planeamiento y la puesta en práctica de las acciones diseñadas y previstas, así como la flexibilidad para adecuarlas han quedado en el Mincetur, como guía y modelo. Todo ello ha sido usado durante el hasta ahora los procesos exitosos de negociaciones comerciales que se han llevado a cabo después de la del TLC con EE.UU. Este modelo, sin embargo, ha sido actualizado y adecuado de manera pragmática a las cambiantes circunstancias de cada uno de los acuerdos negociados con posterioridad. También han sido adecuados y perfeccionados en lo que concierne a acciones y actividades concretas, manteniendo sin cambios los aspectos fundamentales de este diseño.

Este éxito ha sido triple. Por un lado con los socios andinos y EE.UU. Por otro lado, con los actores domésticos, tanto públicos como privados. Y finalmente, con la sociedad civil y la población en general. Con los socios andinos, el Perú demostró liderazgo e intención clara de generar consensos para negociar de manera unificada. Con EE.UU., el Perú demostró capacidad de negociar con solvencia y profesionalismo para llegar de manera pragmática a acuerdos; así como demostrar la seriedad y seguridad de que su política de economía de mercado y de libre comercio era sólida y de largo plazo. Con los actores domésticos públicos y privados el Mincetur demostró capacidad de liderar las negociaciones, coordinar y lograr posiciones nacio- 
nales frente a los disimiles, y algunas veces directamente contrapuestos intereses y objetivos, pudiendo llegar a consensos aceptables para todos. Con la sociedad civil y la población en general, se demostró que las negociaciones se realizaban de manera transparente y que se tenían muy presentes los intereses y el bienestar nacionales.

Ello permitió, primero la aprobación del acuerdo y su modificatoria por parte del Congreso. Segundo, la aprobación y apoyo a la ratificación de parte de casi todos los actores privados. Y tercero, la aprobación del TLC por buena parte de la población.

Más aún, causa satisfacción en el Mincetur que los beneficios propalados durante las negociaciones y en el proceso de ratificación, hayan sino no solo se hayan hecho realidad, sino también, en muchos casos, superados. Por otro lado, para alivio de todos, las preocupaciones en relación a los efectos negativos o no se han hecho realidad o han sido mínimos. Al respecto, hasta el momento, no existe una opinión mayoritariamente contraria al TLC con EE.UU., ni tampoco, vale la pena mencionar, contra ningún otro acuerdo de libre comercio vigente.

Este éxito negociador, sirvió además como un seguro primer eslabón orientado a ofrecer nuevas oportunidades para aumentar las exportaciones no tradicionales peruanas. Lo cual da origen a la multitud de efectos positivos y a algunos negativos. Los primeros deben maximizarse principalmente a través de acciones domesticas de mejoras en la facilitación del comercio. Los segundos se deben superar a través de medidas y mecanismo compensatorios y preventivos internos. Sobre ello, el gobierno siempre ha estado dispuesto a considerar las medidas si fuese el caso.

Perú, gracias en parte, al bien negociado TLC con EE.UU., ha podido continuar con sus negociaciones comerciales internacionales. Ellas, permiten, hasta ahora, el acceso con aranceles preferenciales a mercados de cincuentaitrés países, cubriendo más del noventa por ciento de las exportaciones.

En parte, gracias a estos éxitos de negociación participativa, se ha logrado también el objetivo de hacer de las negociaciones comerciales internacionales y del comercio exterior elementos importantes de la agenda de la política económica nacional.

Por otro lado, también se ha conseguido que todos los actores, públicos y privados, relevantes y relacionados con el quehacer del comercio exterior y la población en general acepten las negociaciones comerciales internacionales como una actividad regular del gobierno y del Estado peruano, sin que las negociaciones causen polémicas ni críticas negativas. Más importante aún, es el apoyo generalizado a las mismas, y la seguridad y confianza que sus efectos son generalmente positivos y beneficiosos para el país, contribuyendo así a mejorar el bienestar nacional. 
Los sucesivos gobiernos han continuado con la utilización de las negociaciones comerciales internacionales, ya que han considerado que logran resultados provechosos para el país. Ello ha permitido, a su vez, el fortalecimiento institucional del Mincetur.

A su vez y/o de manera concomitante con el TLC con EE.UU., el Mincetur, puede contar con una planta de funcionarios de alto nivel profesional. Ellos están regularmente apoyados con el fortalecimiento de sus capacidades en las áreas de sus especializaciones. Los funcionarios, son además, en su mayoría, jóvenes muy comprometidos con su labor profesional, conscientes de que defender los intereses nacionales son fundamentales en su quehacer laboral, realizando sus actividades con una gran mística, sentido de ética y con la convicción de que están haciendo historia y más importante aún, que están haciendo Patria.

\section{Referencias}

Chan, J. (1996). The Politics of Economic Integration in Latin America: A Case Study of the Andean Group, 1969-1995 (tesis de doctorado en Ciencia Política). The City University of New York, EE.UU.

Chan, J. (1997). Aspectos políticos del proceso de integración del Grupo Andino: 1989-1996. Comercio Exterior, 47(5), 378-385.

Chan, J. (2008). APEC y el Perú. Guía sobre el foro de Cooperación Económica Asia-Pacifico y la participación del Perú. 2da. edición. Lima: Universidad de Lima, Fondo Editorial.

Cuarta Cumbre de las Américas (2005). Declaración de Mar del Plata. Mar del Plata, Argentina. Recuperado de http://www.summit-americas.org/Documents\%20for\%20 Argentina\%20Summit\%202005/IV\%20Summit/Declaracion/Declaracion\%20IV\%20 Cumbre\%20de\%20las\%20Americas\%20-\%20Espa\%C3\%B1ol.pdf

Cumbre Extraordinaria de las Américas. (2004). Declaración de Nuevo León. Nuevo León, México. Recuperado de http://www.summit-americas.org/SpecialSummit/Declarations/ Declaration\%20of\%20Nuevo\%20Leon\%20-final-span.pdf >>.

Del Prado, J. (2015). Política exterior del Perú en la Comunidad Andina. En F. Novak y J. García Díaz (Coords.), La política exterior peruana en el siglo XXI. Agenda y propuestas (pp. 159-174). Lima: Instituto de Estudios Internacionales (IDEI) de la Pontificia Universidad Católica del Perú y Konrad Adenauer Stiftung (KAS).

Dingemans, A. (2013). Construyendo estrategias comerciales en América Latina contemporánea. La descuidada dimensión política del comercio internacional. Revista Enfoques, 9(18), 129-152.

Ferrero, A. (2005). Presentación. En Mincetur, TLC Perú-Estados Unidos: nociones clave. Lima: Mincetur, Unidad de Comunicaciones para el TLC Perú-Estados Unidos.

Ferrero, A. (2010). Historia de un desafío. TLC: A la conquista de Estados Unidos y el mundo. Lima: Planeta. 
Ferrero, A. (2013). Los TLC: punto de quiebre para el Perú. En L. León, Acuerdos comerciales del Perú (pp. 121-123). Lima: Congreso de la República del Perú.

Ferreyros, E. (2013). Importancia de los TLC y Acuerdos Comerciales para el Perú. En L. León, Acuerdos comerciales del Perú (pp. 119-120). Lima: Congreso de la República del Perú.

García, J. (2012). La política de comercio exterior y de desarrollo del Perú (1950-2011). En IDEI (Ed.), Veinte años de politica exterior peruana (1991-2011). Libro homenaje por el vigésimo aniversario del Instituto de Estudios Internacionales (IDEI) de la Pontificia Universidad Católica del Perú (pp. 423-453). Lima: Fondo Editorial e Instituto de Estudios Internacionales (IDEI) de la Pontificia Universidad Católica del Perú.

García, J. (2015). Los acuerdos comerciales, su implementación y aprovechamiento. En F. Novak y J. García (Coords.), La politica exterior peruana en el siglo XXI. Agenda y propuestas (pp. 135-153). Lima: Instituto de Estudios Internacionales (IDEI) de la Pontificia Universidad Católica del Perú y Fundación Konrad Adenauer (KAS).

Guillén, M. (2004). Periodismo y exportaciones: el caso de la Comisión para la Promoción de las Exportaciones (PROMPEX). (Informe Profesional de Licenciatura). Universidad Nacional Mayor de San Marcos, Lima, Perú. Recuperado de http://sisbib.unmsm.edu. pe/bibvirtualdata/Tesis/Human/guillen_am/guillen_am.pdf

Milet, P. (2016). Chile y Perú frente a los nuevos desafíos regionales. En P. Milet y F. Novak (Eds.), Desafios en la relación entre Chile y el Perú (pp. 77-90). Lima: Instituto de Estudios Internacionales (IDEI) de la Pontificia Universidad Católica del Perú, Instituto de Estudios Internacionales (IEI) de la Universidad de Chile y Fundación Konrad Adenauer (KAS).

Mincetur - Ministerio de Comercio Exterior y Turismo del Perú. (s/f). TLC Perú - EE.UU. Sobre el acuerdo. Recuperado de http://www.acuerdoscomerciales.gob.pe/index. php?option=com_content\&view=category\&layout=blog\&id=56\&Itemid=79

Mincetur - Ministerio de Comercio Exterior y Turismo del Perú. (s/fa). TLC Perú - EE.UU. Preguntas frecuentes. Recuperado de http://www.acuerdoscomerciales.gob.pe/index. php?option=com_content\&view=category\&layout=blog\&id=59\&Itemid=82

Mincetur - Ministerio de Comercio Exterior y Turismo del Perú. (2005). Las mipymes frente al reto del TLC. Construcción de una agenda de competitividad desde las bases empresariales. Lima: Mincetur. Recuperado de http://www.acuerdoscomerciales.gob.pe/images/stories/ EE.UU./docs/estudios/MIPYMES_FRENTE_TLC.pdf

Mincetur - Ministerio de Comercio Exterior y Turismo del Perú. (2005a). TLC Perú-Estados Unidos: nociones clave. Lima: Mincetur, Unidad de Comunicaciones para el TLC Perú Estados Unidos. Recuperado de http://www.acuerdoscomerciales.gob.pe/images/stories/ EE.UU./docs/estudios/TLC_NOCIONES_CLAVE.pdf

Mincetur - Ministerio de Comercio Exterior y Turismo del Perú. (2005b). La oportunidad de hoy. Tratado de Libre Comercio entre el Perú y Estados Unidos. Lima: Mincetur. Recuperado de http://www.acuerdoscomerciales.gob.pe/images/stories/EE.UU./docs/estudios/TLC_ OPORTUNIDAD_HOY.pdf

MINCETUR - Ministerio de Comercio Exterior y Turismo del Perú. (2005c). TLC y Agro. Todo lo que el productor y el trabajador agrario deben saber acerca del TLC. Recuperado de http:// 
www.acuerdoscomerciales.gob.pe/images/stories/EE.UU./docs/estudios/TLC_AGRO. pdf

MRE - Ministerio de Relaciones Exteriores del Perú (s/f). El Perú y las Américas. Recuperado de http://www.rree.gob.pe/SitePages/politica.aspx\#americas

Mindreau, M. (2005). Del GATT a la OMC (1947-2005). Lima: Universidad del Pacífico.

Palma, H. (2015). Los retos en materia de seguridad. En F. Novak y J. García (Coords.), La politica exterior peruana en el siglo XXI. Agenda y propuestas (pp. 295-310). Lima: Instituto de Estudios Internacionales (IDEI) de la Pontificia Universidad Católica del Perú y Konrad Adenauer Stiftung (KAS).

Parodi, C. (2003). Perú 1960-2000: politicas económicas y sociales en entornos cambiantes. Lima: Centro de Investigación de la Universidad del Pacífico.

Parodi, C. (2016). Perú 1995-2012: cambios y continuidades. Lima: Universidad del Pacífico.

Ramacciotti, B. y Méndez, M. (2012). Las relaciones entre Perú y Estados Unidos: dos décadas de asociación convergente. En IDEI (Ed.), Veinte años de política exterior peruana (19912011). Libro homenaje por el vigésimo aniversario del Instituto de Estudios Internacionales (IDEI) de la Pontificia Universidad Católica del Perú (pp. 95-140). Lima: Fondo Editorial e Instituto de Estudios Internacionales (IDEI) de la Pontificia Universidad Católica del Perú.

Sheahan, J. (2001). La economía peruana desde 1950. Buscando una sociedad mejor. Lima: Instituto de Estudios Peruanos.

Thorp, R. y Bertrasm, G. (1988). Perú: 1890-1977. Crecimiento y politicas en una economía abierta. Lima: Mosca Azul Editores.

Fecha de recepción: 16 de marzo de 2018

Fecha de aprobación: 15 de junio de 2018 